\title{
Constraints on the topology of the Universe from the 2-year COBE data
}

\author{
Angélica de Oliveira Costa ${ }^{1,2}$ and George F. Smoot ${ }^{1}$ \\ E-mail: Angelica@astmag.lbl.gov,Smoot@astmag.lbl.gov
}

(1) Lawrence Berkeley Laboratory, Space Sciences Laboratory and Center for Particle Astrophysics, Building 50-205, University of California, Berkeley, CA 94720.

(2) Instituto Nacional de Pesquisas Espaciais (INPE), Astrophysics Division, São José dos Campos, São Paulo 12227-010, Brazil.

\begin{abstract}
The cosmic microwave background (CMB) is a unique probe of cosmological parameters and conditions. There is a connection between anisotropy in the CMB and the topology of the Universe. Adopting a universe with the topology of a 3-Torus, or a universe where only harmonics of the fundamental mode are allowed, and using 2-years of COBE/DMR data, we obtain constraints on the topology of the Universe. Previous work constrained the topology using the quadrupole component and the correlation function of the CMB. In this letter, we obtain more accurate results by using all multipole moments, avoiding approximations by computing their full covariance matrix. We obtain the best fit for a cubic toroidal universe of scale $7200 \mathrm{~h}^{-1} \mathrm{Mpc}$ for $n=1$. The data set a lower limit on the cell size of $4800 \mathrm{~h}^{-1} \mathrm{Mpc}$ at $95 \%$ confidence and $6000 h^{-1} \mathrm{Mpc}$ at $65 \%$ confidence. These results show that the most probable cell size would be around 1.2 times larger than the horizon scale, implying that the 3 -Torus topology is no longer an interesting cosmological model.
\end{abstract}

Subject headings: cosmology, topology, cosmic microwave background. 


\section{Introduction}

One of the basic assumptions in modern cosmology, the Cosmological Principle, is that on large-scale average our Universe is spatially homogeneous and isotropic. The apparent isotropy on large scales is normally explained as a consequence of spatial homogeneity, in turn understood as natural result of an "inflationary" period of the early universe (see e.g. Kolb and Turner, 1990). An alternative approach to explaining the apparent homogeneity is to assume an expanding universe with small and finite space sections with a non-trivial topology (Ellis and Schreiber, 1986), the "small universe" model.

The "small universe", as its name suggests, should be small enough that we have had time to see the universe around us many times since the decoupling. The topology of the spatial sections can be quite complicated (Ellis, 1971); however, it is possible to obtain small universe models that reproduce a Friedmann-Lemaitre model by choosing certain simple geometries. For example, choosing a rectangular basic cell with sides $L_{x}, L_{y}$ and $L_{z}$ and with opposite faces topologically connected, we obtain a toroidal topology for the small universe known as Torus $T^{3}$. The never-ending repetition of this $T^{3}$ basic cell should reproduce, at least locally, the Friedmann-Lemaitre universe model with zero curvature.

The small universe model has received considerable attention in the past few years, since the topology of the Universe is becoming an important problem for cosmologists. From the theoretical point of view, it is possible to have quantum creation of the Universe with a nontrivial topology, i.e., a multiply-connected topology (Zel'dovich and Starobinsky, 1984). From the 
observational side, this model has been used to explain "observed" periodicity in the distributions of quasars (Fang and Sato, 1985) and galaxies (Broadhurst et al., 1990).

There are four known approaches for placing lower limits on the cell size of the $T^{3}$ model. The first two methods constrain the parameter $R$, an average length scale of the small universe, defined as $R=\left(L_{x} L_{y} L_{z}\right)^{1 / 3}$. The third and fourth methods constrain the parameter $L / y$, the ratio between the cell size $L$, here defined as $L=L_{x}=L_{y}=L_{z}$, and radius of the decoupling sphere $y$, where $y \equiv 2 c H_{o}^{-1}$. The first method constrains $R$ assuming that it is larger than any distinguishable structure. Using this method, Fairall (1985) suggests that $R>500 \mathrm{Mpc}$. The second method constrains $R$ based on "observed" periodicity in quasar redshifts. Attempting to identify opposite pairs of quasars, Fang and Liu (1988) suggested that $R>400 h^{-1} \mathrm{Mpc}$ and using quasar redshift periodicity, Fang and Sato (1985) suggested $R>600 h^{-1} \mathrm{Mpc}$. The third and fourth methods constrain $L / y$ using the CMB. With the third method, Stevens et al. (1993) obtain the constraint $L / y=0.4$ using the quadrupole anisotropy detected in the 1st year of COBE/DMR data (Smoot et al., 1992) while, with the fourth method, Jing and Fang (1994) obtain a best fit $L / y \approx 0.6$ using the correlation function from the 2-year COBE/DMR. Note that these authors defined $y \equiv c H_{o}^{-1}$ instead of $y \equiv 2 c H_{o}^{-1}$.

As pointed out by Zel'dovich (1973), the power spectrum of density perturbations is continuous (i.e., all wave numbers are possible) if the Universe has a Euclidean topology and discrete (i.e., only some wave numbers are pos- 
sible) if the topology has finite space sections. Many years later these ideas were related with the expected CMB power spectrum (Fang and Mo, 1987; Sokolov, 1993; Starobinsky, 1993), mainly after the quadrupole component had been detected by COBE/DMR.

The goal of this letter is to place new and accurate limits on the cell size of a small universe using the harmonic decomposition technique to obtain the data power spectrum (Górski, 1994) and the likelihood technique (Górski, 1994; Bunn and Sugiyama, 1994) to constrain $L / y$. The method that we use to constrain the parameter $L / y$ is quite different from previous work. The method adopted by Stevens et al. (1993) constrains the cell size based in the power spectrum of the $\mathrm{CMB}$; they graphically compare the power spectrum of the standard model with the power spectrum expected for the small universe normalizing to the quadrupole component. Jing and Fang (1994) adopt an different approach: they constrain the cell size using the correlation function of the $\mathrm{CMB}$ and making the approximation that bins of the correlation function are uncorrelated. Our analysis, however, is exact. We compute the full covariance matrix for all multipole components and use this covariance matrix to make a $\chi^{2}$ fit of the power spectrum extracted from the 2 years of COBE/DMR data to the power spectrum expected for a small universe with different cell sizes $L$. For simplicity, we limit our calculation to the case of a $T^{3}$ cubic universe. We present, in the next sections, a description of the power spectrum expected in a $T^{3}$ cubic universe model and the likelihood technique used to constrain $L / y$. 


\section{Power spectrum of the $T^{3}$ universe model}

If the density fluctuations are adiabatic and the Universe is spatially flat, the Sachs-Wolfe fluctuations in the CMB are given by

$$
\frac{\delta T}{T}(\theta, \phi)=-\frac{1}{2} \frac{H_{o}^{2}}{c^{2}} \sum_{\mathbf{k}} \frac{\delta_{\mathbf{k}}}{k^{2}} e^{i \mathbf{k} \cdot \mathbf{x}}
$$

(Peebles, 1982), where $\mathbf{x}$ is a vector with length $y \equiv 2 c H_{o}^{-1}$ that is pointed in the direction of observation $(\theta, \phi), H_{0}$ is the Hubble constant (written in this letter as $100 \mathrm{~h} \mathrm{~km} / \mathrm{s} / \mathrm{Mpc}$ ) and $\delta_{\mathrm{k}}$ is the density fluctuation in Fourier space with the sum taken over all wave numbers $\mathbf{k}$.

It is customary to expand the CMB anisotropy in spherical harmonics

$$
\frac{\delta T}{T}(\theta, \phi)=\sum_{l=0}^{\infty} \sum_{m=-l}^{l} a_{l m} Y_{l m}(\widehat{\mathbf{x}}),
$$

where $a_{l m}$ are the spherical harmonic coefficients and $\widehat{\mathbf{x}}$ is the unit vector in direction $\mathbf{x}$. The coefficients $a_{l m}$ are given by

$$
a_{l m}=-2 \pi i^{l} \frac{H_{o}^{2}}{c^{2}} \sum_{\mathbf{k}} \frac{\delta_{\mathbf{k}}}{k^{2}} j_{l}(k y) Y_{l m}^{*}(\hat{\mathbf{k}}),
$$

where $j_{l}$ are spherical Bessel functions of order $l$. If we assume that the CMB anisotropy is a Gaussian random field, the coefficients $a_{l m}$ are independent Gaussian random variables with zero mean and variance

$$
\left\langle\left|a_{l m}\right|^{2}\right\rangle=16 \pi \sum_{\mathbf{k}} \frac{\left|\delta_{\mathbf{k}}\right|^{2}}{(k y)^{4}} j_{l}^{2}(k y)
$$

(Fang and Mo, 1987; Stevens et al., 1993). Assuming a power-law power spectrum with shape $P(k)=\left|\delta_{k}\right|^{2}=A k^{n}$, where $A$ is the amplitude for 
scalar perturbations and $n$ the spectral index, it is possible to perform the sum in (4) and to obtain

$$
\left\langle\left|a_{l m}\right|^{2}\right\rangle=C_{2} \frac{\Gamma\left(\frac{9-n}{2}\right)}{\Gamma\left(\frac{3+n}{2}\right)} \frac{\Gamma\left(l+\frac{n-1}{2}\right)}{\Gamma\left(l+\frac{5-n}{2}\right)}
$$

(see e.g. Bond and Efsthatiou, 1987). In the literature, the average over the canonical ensemble of universes $\left\langle\left|a_{l m}\right|^{2}\right\rangle$ is usually denoted by

$$
C_{l} \equiv\left\langle\left|a_{l m}\right|^{2}\right\rangle
$$

where the power spectrum $C_{l}$ is related to the rms temperature fluctuation by $\left\langle|\delta T / T|_{r m s}^{2}\right\rangle \equiv \sum_{l}(2 l+1) C_{l} / 4 \pi$.

Note that in a Euclidean topology the Sachs-Wolfe spectrum $C_{l}$ is an integral over the power spectrum; however, in the $T^{3}$ universe this is not the case. In this model, only wave numbers that are harmonics of the cell size are allowed. We have a discrete $\mathrm{k}$ spectrum

$$
\mathrm{k}^{2}=\sum_{i=1}^{3}\left(\frac{2 \pi}{L_{i}}\right)^{2} p_{i}^{2}
$$

(Sokolov, 1993), where $L_{1}, L_{2}$ and $L_{3}$ are the dimensions of the cell and $p_{i}$ are integers. For simplicity, assuming $L=L_{x}=L_{y}=L_{z}$ and the same power-law power spectrum cited before, eq. (4) can be written as

$$
\left\langle\left|a_{l m}\right|^{2}\right\rangle=\frac{16 \pi A}{y^{n}} \sum_{p_{x}} \sum_{p_{y}} \sum_{p_{x}}\left(\frac{L}{2 \pi y p}\right)^{4-n} j_{l}^{2}\left(\frac{2 \pi y p}{L}\right)
$$

where $p^{2}=p_{x}^{2}+p_{y}^{2}+p_{z}^{2}$. According to (8), the th multipole of the CMB temperature is function of the ratio $L / y$. It shows that the more multipole 
components we use in our fit, the stronger our constraints on the cell size will be. However, we cannot use an infinite number of multipole components. The maximum number of multipole components, $l_{\max }$, will be limited by two things: the limit where the map is noise dominated and the limit where we can truncate the Fourier series without compromising the harmonic decomposition technique (see Górski, 1994).

Using eq. (8), we calculated the expected power spectrum for a $T^{3}$ universe with different cell sizes $L / y$ from 0.1 to $3.0, n=1$ and $l_{\max }=30$, where $l_{\max }=30$ is the limit at which we truncate our data power spectrum. In Figure 1, we plot $l(l+1) C_{l}$ versus $l$ and normalize all values to the last multipole component $l=30$. Note that for very small cells $(L \ll y)$, the low order multipoles are suppressed. The power spectrum for small cells (as $L / y=0.1,0.5$ or 1.0) shows the presence of "bumps" that disappear as the cell size increases $(L / y \gtrsim 1.5)$. The power spectrum finally becames flat for large cell sizes $(L / y \gtrsim 3.0)$. These "bumps" can be explained if we remember that only the harmonics of the cell size are allowed to be part of the sum in (8). When the cell size is small there are fewer modes of resonance, and no modes larger than the cell size appear in the sum in (8). As the cell size increases, the sum approaches an integral and the $T^{3}$ power spectrum becomes flat.

In this letter we restrict our analysis to $n=1$. This assumption, however, does not weaken our results since the $T^{3}$ model with other $n$-values tends to fit the data as poorly as with $n=1$. For instance, we obtain the maximum likelihood at the same ratio $L / y$ for $n=1$ and $n=1.5$. This happens 
because the "bumps", and not the overall slope, are responsible for the disagreement between the model and the data.

\section{Data Analysis}

Each DMR sky map is composed of 6144 pixels and each pixel $i$ contains a measurement of the sky temperature at position $\mathbf{x}_{i}$. Considering that the temperatures are smoothed by the DMR beam and contaminated with noise, the sky temperatures are described by

$$
\left(\frac{\delta T}{T}\right)_{i}=\sum_{l m} a_{l m} B_{l} Y_{l m}\left(\widehat{\mathbf{x}}_{i}\right)+n_{i}
$$

where $B_{l}$ is the DMR beam pattern and $n_{i}$ is the noise in pixel $i$. We use the values of $B_{l}$ given by Wright et al. (1994a), which describes the actual beam pattern of DMR horns, a non perfect gaussian beam. We model the quantities $n_{i}$ in (9) as Gaussian random variables with mean $\left\langle n_{i}\right\rangle=0$ and variance $\left\langle n_{i} n_{j}\right\rangle=\sigma_{i}^{2} \delta_{i j}$, assuming uncorrelated pixel noise (Lineweaver et al., 1994).

When we have all sky coverage, the $a_{l m}$ coefficients are given by

$$
a_{l m}=\int_{4 \pi}\left(\frac{\delta T}{T}\right) Y_{l m}^{*}(\hat{\mathbf{x}}) d \Omega .
$$

In the real sky maps, we do not have all sky coverage. Because of the uncertainty in Galaxy emission, we are forced to remove all pixels between $20^{\circ}$ under and above the Galaxy plane. This cut represents a loss of almost $34 \%$ of all sky pixels and destroys the orthogonality of the spherical har- 
monics. Replacing the integral in (10) by a sum over the number of pixels that remain in the sky map after the Galaxy cut, $N_{p i x}$, we define a new set of coefficients by

$$
b_{l m} \equiv w \sum_{i=1}^{N_{p i x}}\left(\frac{\delta T}{T}\right)_{i} Y_{l m}^{*}\left(\hat{\mathbf{x}}_{i}\right)
$$

where the normalization is chosen to be $w \equiv 4 \pi / N_{p i x}$. Substituting (9) into (11), we obtain

$$
b_{l m}=\sum_{l_{1} m_{1}} a_{l_{1} m_{1}} B_{l_{1}} W_{l_{1} m m_{1}}+w \sum_{i=1}^{N_{p i x}} n_{i} Y_{l m}^{*}\left(\widehat{\mathbf{x}}_{i}\right)
$$

with covariance

$$
\begin{aligned}
\left\langle b_{l m} b_{l^{\prime} m^{\prime}}^{*}\right\rangle & =\sum_{l_{1} m_{1}} W_{l l_{1} m m_{1}} W_{l^{\prime} l_{1} m^{\prime} m_{1}} C_{l_{1}} B_{l_{1}}^{2}+ \\
& +w^{2} \sum_{i=1}^{N_{p i x}} \sigma_{i}^{2} Y_{l m}^{*}\left(\widehat{\mathbf{x}}_{i}\right) Y_{l^{\prime} m^{\prime}}\left(\hat{\mathbf{x}}_{i}\right)
\end{aligned}
$$

where

$$
W_{l l_{1} m_{1}} \equiv w \sum_{i=1}^{N_{p i x}} Y_{l m}^{*}\left(\widehat{\mathbf{x}}_{i}\right) Y_{l_{1} m_{1}}\left(\widehat{\mathbf{x}}_{i}\right)
$$

Defining our multipole estimates as

$$
C_{l}^{D M R} \equiv \frac{1}{2 l+1} \sum_{m} b_{l m} b_{l m}^{*}
$$

their expectation values are simply

$$
\left\langle C_{l}^{D M R}\right\rangle \equiv \frac{1}{2 l+1} \sum_{m}\left\langle b_{l m} b_{l m}^{*}\right\rangle
$$


and their covariance matrix $M$ is given by

$$
M_{l^{\prime}} \equiv \frac{2}{(2 l+1)\left(2 l^{\prime}+1\right)} \sum_{m m^{\prime}}\left\langle b_{l m} b_{l^{\prime} m^{\prime}}^{*}\right\rangle^{2} .
$$

The $C_{l}^{D M R}$ coefficients are not good estimates of the true multipole moments $C_{l}$. However, they are useful for constraining our cosmological parameters. The likelihood and the $\chi^{2}$ are, respectively, defined by

$$
-2 \ln \mathcal{L}=\chi^{2}+\ln |\mathrm{M}|
$$

and

$$
\chi^{2} \equiv \mathbf{C}^{T} \mathbf{M}^{-1} \mathbf{C}
$$

where $C^{T}$ and $C$ are $l_{\text {max }}$-dimensional row and column vectors with entries $C_{l}=\widehat{C}_{l}^{D M R}-\left\langle C_{l}^{D M R}\right\rangle$ and $\mathrm{M}$ is the covariance matrix as described in (17) with dimension $l_{\max } \times l_{\max }$. Here $\widehat{C}_{l}^{D M R}$ denotes the $C_{l}^{D M R_{-}}$-coefficients actually extracted from the data.

Because the perturbations depend on an unknown constant $A$, the power spectrum normalization, we have to constrain two parameters at once. In practice, this calculation is done by fixing the ratio $L / y$ and changing the normalization by a small factor. We multiply the first term on the right side of (13) by this factor and calculate a new covariance matrix. Repeating this procedure for each cell size we, finally, get a likelihood grid that constrains the ratio $L / y$ and the normalization parameter. 


\section{Results}

In Figure 2, we show the angular power spectrum $\hat{C}_{l}^{D M R}$ extracted from the data. We use a 2-year combined 53 plus $90 \mathrm{GHz}$ map, with Galaxy cut of $20^{\circ}$, monopole and dipole removed. We plot $l(l+1) \hat{C}_{l}^{D M R}$ versus $l$ from $l=2$ to $l=30$, with bias $\left(\left\langle C_{l}^{D M R}\right\rangle-C_{l}\right)$ removed and error bars given by the diagonal ierms of the covariance matrix $M$. In computing the bias and error bars, we assume eq. (5) with $n=1$. The shape of this power spectrum and its multipole values are consistent with values reported by Wright et al. (1994b), and for $l>15$ the power spectrum is basically dominated by noise.

We computed the likelihood function $\mathcal{L}\left(L / y, \sigma_{7^{\circ}}\right)$, using it to constrain the ratio $L / y$ and the normalization $\sigma_{7^{\circ}}$, where $\sigma_{7^{\circ}}$ is the rms variance at $7^{\circ}$. For the data set described above, we found maximum likelihood at $\left(L / y, \sigma_{7^{\circ}}\right)=(1.2,37.4 \mu K)$. In Figure 3 , we plot the likelihood function $\mathcal{L}\left(L / y, \sigma_{7^{\circ}}\right)$. Notice that the likelihoods cannot be normalized because they do not converge to zero for very large cell sizes, i.e., the volume under the likelihood function is infinite. Since the likelihoods are not zero for very large cell sizes, we could naively consider that the probability of the universe being small is essentially zero. However, this conclusion is clearly exaggerated and based on the fact that we multiplied our likelihoods by a uniform prior, and there is nothing special about adopting a uniform prior. In order to obtain rigorous confidence limits for our analysis, we replace the maximum likelihood fit by a minimum $\chi^{2}$ fit.

We compute the chi-squared function $\chi^{2}\left(L / y, \sigma_{7^{\circ}}\right)$ and use it to constrain the ratio $L / y$ and the normalization $\sigma_{7^{\circ}}$. In Figure 4 , we plot the probability 
that the $T^{3}$ model is consistent with the data as a function of the ratio $L / y$ and the normalization $\sigma_{7^{\circ}}$ (bottom). Confidence limits of $68 \%, 95 \%$ and 99.7\% are shown in the contour plot (top). We found the highest consistency probability $\left(\operatorname{minimum} \chi^{2}\right)$ at $\left(L / y, \sigma_{7^{\circ}}\right)=(1.2,49.7 \mu K)$, represented by a cross in the contour plot. Removing the quadrupole, we obtained similar results, see Table 1 for the lower limits on cell sizes. We obtain the constraint $L / y=1.2_{-0.5}^{+\infty}$ at $95 \%$ confidence. We cannot place an upper limit on the cell size: all large cells are equally probable and have the best fit around $\sigma_{7^{\circ}}=49.7 \mu \mathrm{K}$.

\section{Conclusions}

The strong constraint from our analysis comes from the predicted power spectrum of the $T^{3}$ universe; see Figure 1. According to this plot, a reduction in the cell size to values below the horizon scale should suppress the quadrupole and low multipole anisotropies, while the suppression is negligible if the cell is very large, at least, larger than the horizon. It is possible to notice these properties in Figures 3 and 4: both favor large cell sizes. The observed presence of the quadrupole and other low order anisotropies automatically constrains our cell to be very large. In other words, even before making the $\chi^{2}$ fit, we expect to obtain very large cells.

We remind the reader that our analysis is for $n=1$. We made this assumption because the results of fitting the $T^{3}$ model seem to be insensitive to changes in $n$ and the "bumps", not the overall slope, are responsible for the poor fit between the model and the data. In other words, this assumption 
has nothing to do with inflation.

From the COBE/DMR data, we obtain the best $\chi^{2}$ fit for a toroidal universe with $L / y=1.2$, which corresponds to a cell size of $L=7200 h^{-1} \mathrm{Mpc}$. A cell size below $70 \%$ of the size of the horizon $(L / y<0.7)$ is incompatible with the COBE measurements at $95 \%$ confidence, and a cell size below the size of the horizon $(L / y<1.0)$ is ruled out at $65 \%$ confidence. Since the $T^{3}$ topology is interesting if the cell size is considerably smaller than the horizon, this model loses most of its appeal.

We would like to thank Jon Aymon, Douglas Scott and Max Tegmark for many useful comments and help with the manuscript. AC acknowledges SCT-PR/CNPq Conselho Nacional de Desenvolvimento Científico e Tecnológico for her financial support under process No.201330/93-8(NV). This work was supported in part by the Director, Office of Energy Research, Office of High Energy and Nuclear Physics, Division of High Energy Physics of the U.S. Department of Energy under contract No.DE-AC03-76SF00098. 
Table 1: Lower limits on $\mathrm{L} / \mathrm{y}$

\begin{tabular}{ccc}
\hline Confidence Level & L/y with $C_{2}$ & L/y without $C_{2}$ \\
\hline $65 \%$ & 1.0 & 1.0 \\
$90 \%$ & 0.8 & 0.7 \\
$95 \%$ & 0.7 & 0.6 \\
$99.7 \%$ & 0.6 & 0.6 \\
\hline
\end{tabular}




\section{References}

Bond, J.R. \& Efsthatiou, G. 1987, Mon. Not. R. Astr. Soc., 226:655.

Broadhurst, T.J. et al. 1990, Nature, 343:726.

Bunn, E. \& Sugiyama, N. 1994, preprint (astro-ph/9407069)

Ellis, G.F.R. 1971, Gen. Rel. and Grav., 2(1):7.

Ellis, G.F.R. \& Schreiber, G. 1986, Phys. Lett. A, 115(3):97.

Fairall, A.P. 1985, Mon. Not. R. Astr. Soc. of South. Africa, 44(11):114.

Fang, L.Z. \& Liu, Y.L. 1988, Mod. Phys. Lett. A, 3(13):1221.

Fang, L.Z. \& Mo, H. 1987, Mod. Phys. Lett. A, 2(4):229.

Fang, L.Z. \& Sato, M. 1985, Gen. Rel. and Grav., 17(11):1117.

Górski, K.M. 1994, Ap. J. Lett., 430:L85.

Jing, Y.P. \& Fang, L.Z., 1994, Phy. Rev. Lett., 73(14):1882.

Kolb, E.W. \& Turner, M.S. 1990, The Early Universe, Addison-Wesley.

Lineweaver, C. et al. 1994, preprint (astro-ph/9403021).

Peebles, P.J.E. 1982, Ap. J. Lett., 263:L1.

Smoot, G.F. et al. 1992, Ap. J. Lett., 396:L1.

Sokolov, I.Y. 1993, JETP Lett., 57(10):617.

Starobinsky, A.A. 1993, JETP Lett., 57(10):622.

Stevens, D. et al. 1993, Phy. Rev. Lett., 71(1):20.

Wright, E.L. et al. 1994a, Ap. J., 420:1.

Wright, E.L. et al. 1994b, preprint (astro-ph/9401015).

Zel'dovich, Ya B. 1973, Comm. Astrophys. Space Sci., 5(6):169.

Zel'dovich, Ya B. and Starobinsky, A.A. 1984, Sov. Astron. Lett., 10(3):135. 


\section{Figure Captions}

Figure 1: Expected power spectrum for the $T^{3}$ universe model with $n=1$ for different cell sizes with $L / y$ from 0.1 to 3.0 .

Figure 2: Power spectrum of the 2-year combined $53+90 \mathrm{GHz} \mathrm{COBE} / \mathrm{DMR}$ data with bias removed and the error bars given by the diagonal terms of the covariance matrix $M$.

Figure 3: The likelihood function $\mathcal{L}\left(L / y, \sigma_{7^{\circ}}\right)$ for the $T^{3}$ universe model with $n=1$.

Figure 4: The probability that the $T^{3}$ model is consistent with the data is plotted as a function of the ratio $L / y$ and the normalization $\sigma_{7^{\circ}}$ (bottom). Confidence limits of $68 \%, 95 \%$ and $99.7 \%$ are shown in the contour plot (top). We found the highest consistency probability (minimum $\chi^{2}$ ) at $L / y=$ 1.2 , represented by a cross in the contour plot. 


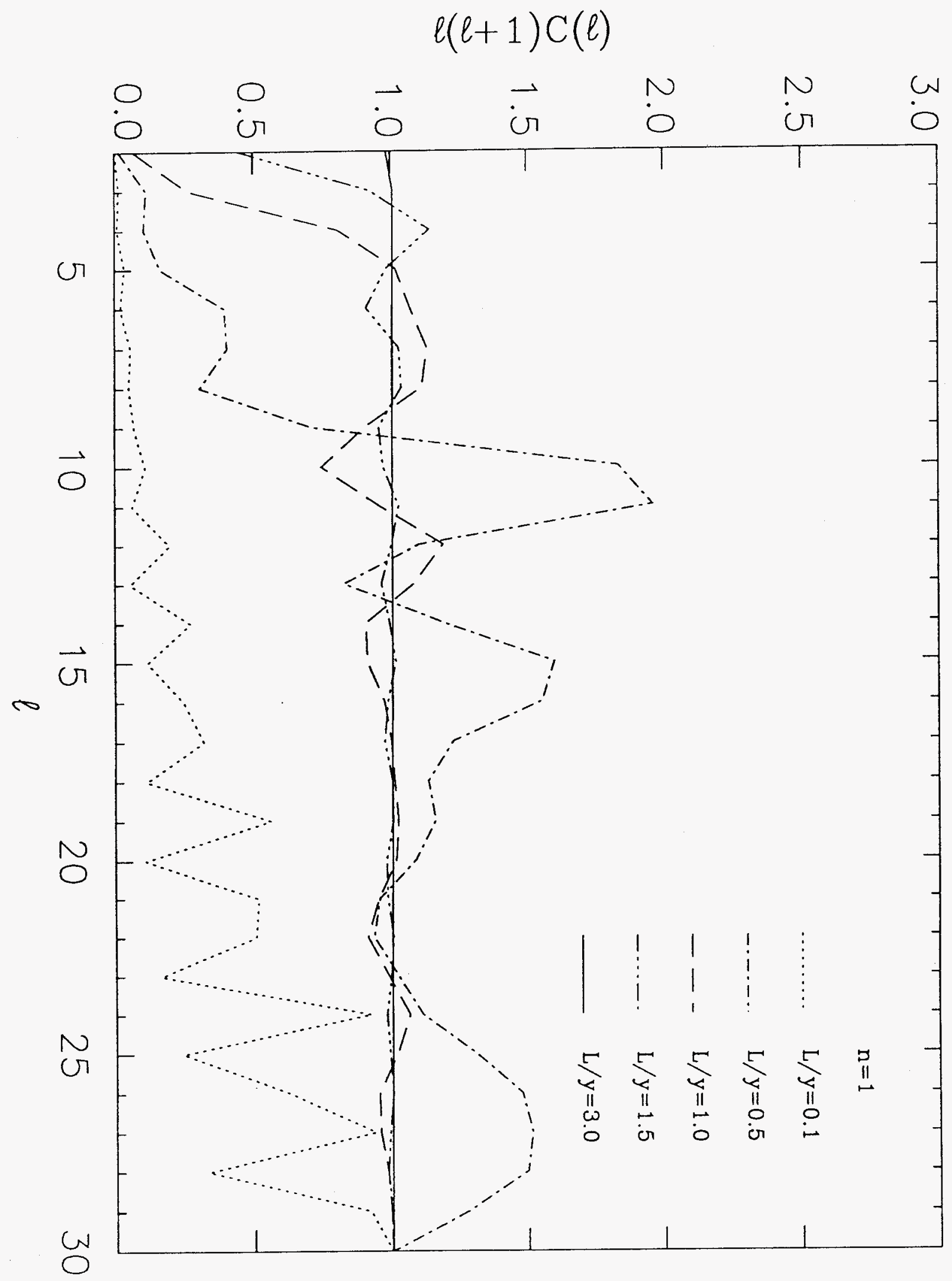




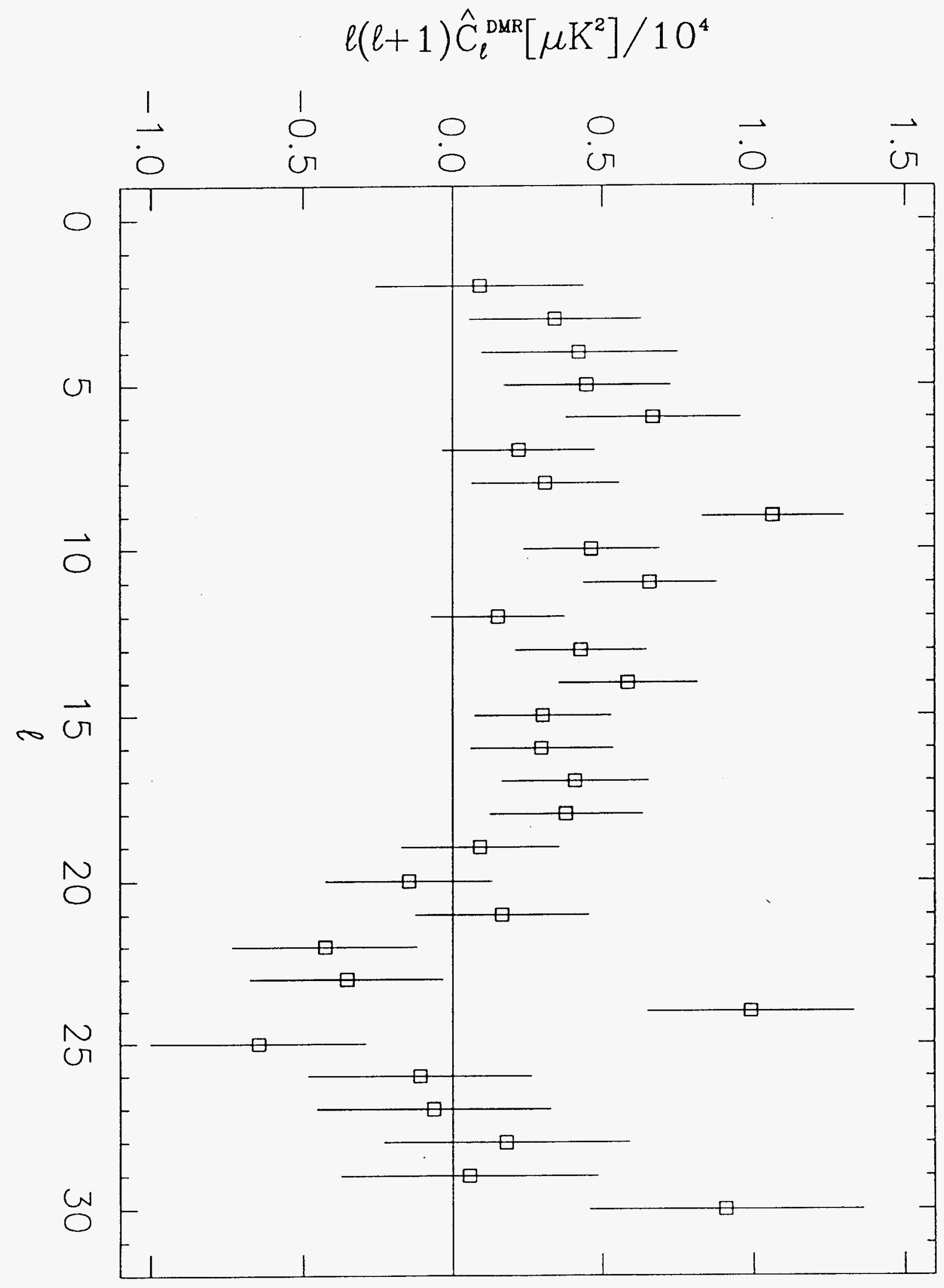




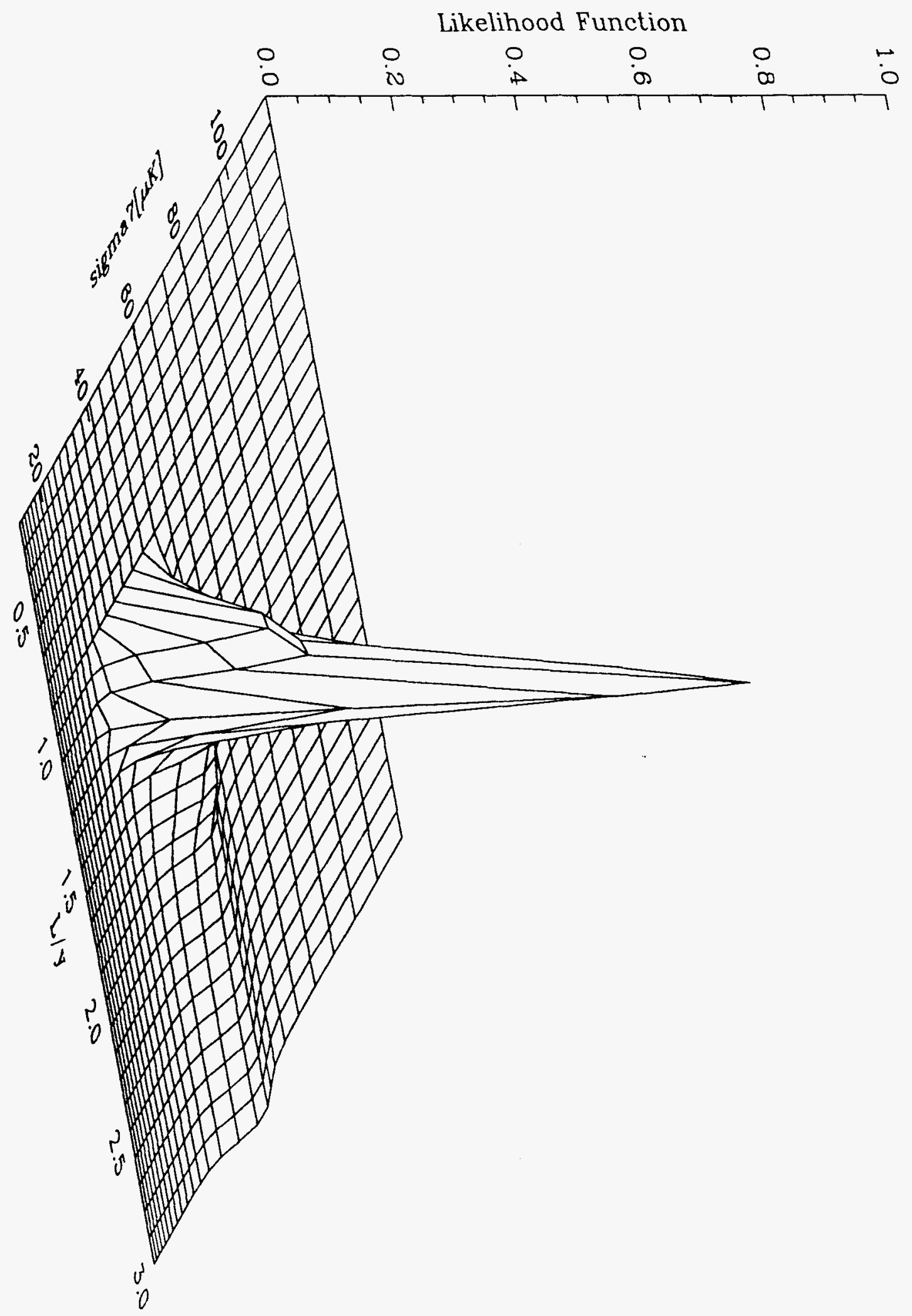




$$
\text { 给 }
$$

